\title{
Role of clevidipine butyrate in the treatment of acute hypertension in the critical care setting: a review
}

REVIEW

\author{
Ahmed S Awad \\ Michael E Goldberg \\ Department of Anesthesiology, \\ Cooper University Hospital, \\ UMDNJ-Robert Wood Johnson \\ Medical School, Camden Campus, \\ Camden, New Jersey, USA
}

This article was published in the following Dove Press journal:

Vascular Health and Risk Management

5 June 2010

Number of times this article has been viewed
Correspondence: Michael E Goldberg Department of Anesthesiology, Cooper University Hospital, UMDNJ-Robert Wood Johnson Medical School, Camden Campus, Camden, NJ 08I03, USA

Tel + I 856-342-2919

Fax + I 856-968-8239

Email goldberg-mike@cooperhealth.edu
Abstract: Acutely elevated blood pressure in the critical care setting is associated with a higher risk of acute end-organ damage (eg, myocardial ischemia, stroke, and renal failure) and perioperative bleeding. Urgent treatment and careful blood pressure control are crucial to prevent significant morbidity. Clevidipine butyrate $\left(\right.$ Cleviprex $\left.^{\mathrm{TM}}\right)$ is an ultrashort-acting, third-generation intravenous calcium channel blocker. It is an arterial-selective vasodilator with no venodilatory or myocardial depressive effects. Clevidipine has an extremely short half-life of approximately 1 minute as it is rapidly metabolized by blood and tissue esterases. These metabolites are then primarily eliminated through urine and fecal pathways. The rapid onset and the short duration of action permit tighter and closer adjustment of the blood pressure than is possible with other intravenous agents.

Keywords: calcium channel blocker, antihypertensive medications, end-organ damage, hypertensive crisis, hypertensive urgency

\section{Introduction}

Systemic hypertension is the most common cardiovascular condition, affecting as many as 1 billion people worldwide and responsible for 1 million deaths. ${ }^{1}$ It affects about 72 million individuals in the United States. ${ }^{2}$ According to American Heart Association estimates, it is responsible for more than 54,000 deaths in 2004 in the US alone. ${ }^{3}$ The incidence of hypertensive emergency is disproportionately higher in the elderly, male, and African American populations. ${ }^{4}$ Approximately $1 \%-2 \%$ of all patients with hypertension are estimated to have hypertensive emergencies. ${ }^{2}$

The Joint National Committee on prevention, detection, evaluation, and treatment of high blood pressure (JNC-7) publication defines hypertensive emergency as a severe elevation in blood pressure (BP) (usually $>180 / 120 \mathrm{mmHg}$ ) complicated by evidence of impending or progressive target organ dysfunction, ${ }^{5}$ such as neurologic changes, hypertensive encephalopathy, cerebral infarction, intracranial hemorrhage, myocardial ischemia or infarction, acute left ventricular dysfunction, acute pulmonary edema, aortic dissection, renal insufficiency, or eclampsia. ${ }^{6}$ Hypertensive urgency is defined as "severe elevations in BP without progressive target organ dysfunction." ${ }^{5}$ One study of emergency department admissions found that hypertensive crises accounted for $27.5 \%$ of all medical emergencies and urgencies in patients arriving at an emergency department, with $77 \%$ having a history of hypertension. ${ }^{7}$ submit your manuscript $\mid$ www.dovepress.com 


\section{Hypertension in critical care and perioperative surgical settings}

In medical intensive care units (ICUs), the epidemiology of hypertensive urgency or hypertensive emergency has not been clearly defined, but appears to be frequent. ${ }^{8}$ Elevated BP during induction of anesthesia, intubation of the airway, emergence from anesthesia, and extubation of the airway can result in hypertensive urgency. Furthermore, operative procedures, including peripheral vascular surgery, coronary artery bypass, carotid endarterectomy, intracranial tumor resection, and aneurysm-clipping procedures, present other critical periods during which the strict control of $\mathrm{BP}$ is mandatory. ${ }^{6,9}$

Preoperative hypertensive emergencies, such as acute aortic dissection, hypertension in pregnancy, and pheochromocytoma, also require aggressive control of $\mathrm{BP}^{6}$ A significant elevation in BP during the immediate postoperative period is defined as acute postoperative hypertension (APH) and may lead to multiple organ or surgical-site complications. APH has been observed within 2 hours after surgery in most cases. ${ }^{10}$ Postoperative hypertension is defined as a systolic blood pressure (SBP) $>190 \mathrm{mmHg}$ and/or diastolic blood pressure (DBP) $>100 \mathrm{mmHg}$ on 2 consecutive readings after surgery. ${ }^{11}$

Acute perioperative hypertension affects up to $80 \%$ of patients undergoing cardiac surgery and up to $25 \%$ of patients undergoing major noncardiac surgery. ${ }^{12}$ Patients with perioperative hypertension commonly have a history of hypertension. Acute hypertension can result in an increase in afterload to the left ventricle, which may cause a reduction in the stroke volume, an elevation in the left ventricular filling pressure, and pulmonary congestion. ${ }^{13}$ The risk of myocardial ischemia, heart failure, stroke, neurocognitive dysfunction, renal failure, and bleeding are increased in patients with perioperative hypertension. ${ }^{14}$ In addition, adrenergic stimulation (occurring before, during, and after the operation) and activation of stress hormones and the renin-angiotensin system in both previously hypertensive and nonhypertensive patients may increase the risk of postoperative crises. ${ }^{8}$

\section{Acute management of hypertensive emergencies and urgencies}

Severely hypertensive patients without end-organ damage may be treated with an oral antihypertensive ${ }^{10}$ within 24 hours to several days in a closely monitored outpatient setting. ${ }^{15}$ Patients with hypertensive emergencies require intensive BP control with intravenous antihypertensives ${ }^{14}$ usually within 1 hour of occurrence. ${ }^{15}$
On the basis of JNC-7 guidelines, the general therapeutic goal is to lower the mean arterial pressure (MAP) by $20 \%-25 \%$ within 60 minutes and, if the patient is then stable, to $160 / 100-110 \mathrm{mmHg}$ over the next 2-6 hours. ${ }^{5}$ Concomitantly, one should consider that an acute lowering of BP will reduce the blood flow to tissue without prompt compensatory vessel dilation, leading to ischemia of endorgan tissue. ${ }^{11}$ Aortic dissection presents a different situation. The guideline followed in this case is to reduce the SBP to at least $120 \mathrm{mmHg}$ within 20 minutes, with commensurate protection against reflex tachycardia. ${ }^{5}$ In APH in cardiac surgery patients, treatment is recommended for a $\mathrm{BP}>140 / 90$ or a $\mathrm{MAP} \geq 105 \mathrm{mmHg}{ }^{16}$

\section{Choice of agent}

Many agents have been used to treat acute, severe elevations of BP, but despite having been used for years, most of these agents are not ideal across the broad range of comorbidities. Intravenous antihypertensive agents available for the management of hypertensive emergencies fall into the broad categories of arterial vasodilators (hydralazine, fenoldopam, nicardipine, clevidipine, and enalaprilat), venous vasodilators (nitroglycerin), mixed venous and arterial vasodilators (sodium nitroprusside), negative inotropic/chronotropic agents with (labetalol) or without vasodilator properties (esmolol), and $\alpha$-adrenergic receptor blockers for increased sympathetic activity (phentolamine).

Intermittent intravenous labetalol is most often selected as the initial intravenous antihypertensive for patients with acute severe hypertension without stroke. ${ }^{17}$ Nicardipine is most often administered initially for patients with acute hemorrhagic stroke followed by intermittent intravenous labetalol and sodium nitroprusside. ${ }^{17}$

The main indications for sodium nitroprusside include hypertensive crises complicated by hypertensive encephalopathy, heart failure, aortic dissection, and adrenergic crisis. ${ }^{15}$ It has a nonselective effect on both arterial and venous vessels, with rapid action within 1-2 minutes. However, hypotension is common, and tachyphylaxis may also develop. Often, rebound hypertension is seen with abrupt withdrawal. ${ }^{18}$ Nitroglycerin is commonly used in hypertensive crises complicated by ischemic heart disease and after coronary bypass. ${ }^{19}$ Its mechanism of action is through dilation of the venous capacitance vessels, which also results in a decrease in preload. ${ }^{13}$ Drug tolerance is a common concern with nitroglycerin and sodium nitroprusside..$^{20}$

Esmolol, a selective $\beta$-antagonist, has a high plasma clearance with a terminal half-life of approximately 10 minutes, 
allowing for titration to the desired level. Esmolol has been commonly used during perioperative hypertension in cardiac surgery. ${ }^{21}$ It can be titrated to the desired effect because of its high plasma clearance and short half-life (approximately 10 minutes). However, administration can result in a decrease in cardiac output $(\mathrm{CO})$ rather than systemic vascular resistance (SVR), which is most commonly the cause of elevated BP during cardiac surgery. ${ }^{20}$

Calcium channel blockers (CCBs) are a heterogeneous class of drugs used in the treatment of hypertension, coronary artery disease (CAD), and dysrhythmias. There are 3 classes of calcium channels blockers: dihydropyridines (eg, nicardipine, clevidipine), phenylalkylamines (eg, verapamil), and benzothiazepines (eg, diltiazem) ${ }^{22}$ Dihydropyridine-type calcium channel antagonists like nicardipine, isradipine, and nifedipine have been used successfully to control BP during cardiac surgery. They have little effect on heart rate without affecting myocardial contractility because their effect is selective for vascular smooth muscle as opposed to the myocardium. They have little activity in the cardiac muscle or the sinoatrial node. ${ }^{23}$

The ideal agent for rapid control of BP would be an arterial-specific vasodilator that has a rapid onset and offset, a low incidence of toxicity, and does not cause reflex tachycardia. ${ }^{24}$ This article reviews the preclinical trials, pharmacologic and clinical efficacy, and tolerability of clevidipine butyrate (Cleviprex $^{\mathrm{TM}}$; The Medicines Company, Parsippany, NJ, USA) and provides insights and evidence to support its clinical applicability in managing emergent hypertensive conditions.

\section{Clevidipine chemistry and formulation}

Clevidipine butyrate is a mixture of 2 enantiomers: S-clevidipine and R-clevidipine. ${ }^{9}$ It is a rapid-acting, dihydropyridine L-type calcium channel antagonist. ${ }^{13}$ These voltage-gated channels are expressed in several excitable cell types, including cardiac and vascular cells, and mediate calcium influx during arterial smooth muscle membrane depolarization. ${ }^{25}$ The loss of extracellular calcium ion influx inhibits intracellular phosphodiesterase, which raises guanosine monophosphate levels, inhibiting vascular smooth muscle contractility, myocardial contractility, and cardiac conduction. ${ }^{6}$

\section{Pharmacologic effects of clevidipine in animal studies}

Clevidipine was found to decrease L-type calcium channel current in guinea pig myocytes, suggesting that its primary mechanism of action is the prevention of transmembrane calcium influx. ${ }^{20}$ Clevidipine was also reported to be an equipotent inhibi- tor of spontaneous myogenic, neurogenic, and agonist-induced contractions of the rat portal vein, further suggesting a common inhibitory pathway. ${ }^{20}$ The effect of clevidipine on spontaneous heart rate was studied in isolated Langendorff-perfused rat hearts and compared with those treated with isradipine and nifedipine. ${ }^{20}$ In contrast to nifedipine and isradipine, clevidipine did not interfere with spontaneous heart rate or atrioventricular conduction. ${ }^{26}$ The antihypertensive potency and the duration of action of clevidipine were compared with other compounds (nitroglycerin, sodium nitroprusside, felodipine, nicardipine, and isradipine) in anesthetized spontaneously hypertensive rats and normotensive rats. The recovery time after clevidipine administration was similar to that of nitroglycerin, longer than that of sodium nitroprusside, and considerably shorter than that of the calcium antagonists felodipine, isradipine, and nicardipine. ${ }^{20}$ The effects of intravenous clevidipine, at increasing doses, on central hemodynamics of anesthetized dogs were compared with those of sodium nitroprusside. Clevidipine reduced mean aortic $\mathrm{BP}$ in a dose-dependent manner by decreasing total peripheral resistance. $\mathrm{CO}$ increased as a result of an increase in stroke volume while heart rate remained unaffected. Sodium nitroprusside decreased BP by decreasing $\mathrm{CO}$ through its venodilatory effect. ${ }^{27}$ The effect of clevidipine on ischemia/reperfusionrelated organ injuries has also been investigated. The possible protective effects of therapeutic and nontherapeutic doses of clevidipine were compared with that of a therapeutic dose of sodium nitroprusside in the rat model of splanchnic ischemia. Clevidipine was found to prevent the deterioration of mesenteric circulation during re-reperfusion after mesenteric ischemia. ${ }^{28}$ Clevidipine was found to significantly reduce infarct size in pigs with myocardial ischemia when administered during the last 10 minutes of ischemia and the first 5 minutes of reperfusion. ${ }^{29}$ Clevidipine was reported to be effective in preserving renal reperfusion function in an experimental rat model of induced acute ischemic renal failure. Either clevidipine or fenoldopam, a dopamine $\mathrm{D}_{1}$-receptor agonist, was infused before reperfusion. Although no significant difference in glomerular filtration was observed between clevidipine and fenoldopam, clevidipine reduced losses in both sodium and water after reperfusion, suggesting that clevidipine may have a protective effect on renal tubular function..$^{30}$ The mechanisms of the protective action of clevidipine on ischemic organs are multifactorial, involving inhibition of oxygen-free radicals, calcium overload, and nitric oxide-related mechanisms. ${ }^{1,20}$

\section{Clinical pharmacology of clevidipine}

Clevidipine has been studied in healthy volunteers, patients with essential hypertension, and in anesthetized or sedated 
patients during and after cardiac surgery. Clevidipine has a rapid onset (2-4 minutes) and offset of action (5-15 minutes). ${ }^{20}$ In cardiac surgery patients, the initial phase half-life is less than 1 minute, and its terminal half-life is approximately 4 minutes. ${ }^{6}$ Clevidipine has exhibited greater inotropic than chronotropic selectivity. ${ }^{31}$ It exerts arterial-specific vasodilatory effects and is characterized by an ultrafast onset and offset of effect in decreasing BP. ${ }^{32}$ The resting potential of vascular smooth muscle cells is less negative than that of the cardiac myocytes, which may partially explain the vascular selectivity of clevidipine. ${ }^{3}$

The ability of clevidipine to control BP in high-risk cardiovascular surgery patients was observed to occur within 1-2 minutes when administered in double-blind, placebocontrolled trials. ${ }^{3,12}$ Clevidipine has minimal effect on venous vascular tone. Because of its selective afterload reduction, clevidipine increases $\mathrm{CO}$ with little influence on left ventricular filling pressure and pulmonary capillary wedge pressure. ${ }^{32}$ Clevidipine may have diuretic properties due to a direct inhibitory effect on renal tubular reabsorption. ${ }^{33}$

In normotensive subjects, clevidipine was observed to cause a reduction in MAP, systemic vascular resistance, and pulmonary vascular resistance as well as an increase in stroke volume, with no change in heart rate or cardiac preload. ${ }^{3}$ During 12 hours of clevidipine infusion, a significant decrease in MAP and SVR was observed in postcardiac surgery patients at doses of $\geq 1.37 \mu \mathrm{g} / \mathrm{kg} / \mathrm{min}$, with no changes in heart rate, cardiac index, or cardiac filling pressure. The half-life remained less than 2 minutes. ${ }^{13}$ Clevidipine has been compared with sodium nitroprusside for the treatment of hypertension after coronary artery bypass graft (CABG) surgery. SVR and heart rate were found to be lower with clevidipine, whereas preload, stroke volume, and pulmonary vascular resistance were higher with clevidipine. ${ }^{12,34}$

\section{Dosage}

\section{Initial dose and titration}

The starting dose of clevidipine is $1-2 \mathrm{mg} / \mathrm{h}$, doubled every 90 seconds until BP approaches the target (SBP reduced by $\geq 15 \%$ ), then increased by less than double every 5-10 minutes as target pressure approaches. ${ }^{35}$ An increase in infusion rate of $1-2 \mathrm{mg} / \mathrm{h}$ generally results in a decrease in SBP of approximately $2-4 \mathrm{mmHg} .^{3}$ In addition, this drug can be safely used as an infusion for as long as 96 hours. ${ }^{2,36}$

\section{Maintenance and maximum dose}

The recommended maintenance dose is $4-6 \mathrm{mg} / \mathrm{h}$ as a continuous intravenous infusion for response in most patients.
In clinical studies, most patients were treated with maximum doses of $16 \mathrm{mg} / \mathrm{h}$, but patients with severe hypertension may require doses up to $32 \mathrm{mg} / \mathrm{h}$ (limited data are available at this dose). ${ }^{37}$ Clevidipine is commercially available in a lipid emulsion. Because of lipid-load restrictions, no more than $1,000 \mathrm{~mL}$ or an average of $21 \mathrm{mg} / \mathrm{h}$ of clevidipine infusion is recommended per 24 hours. ${ }^{37}$ The efficacy and safety of clevidipine in pediatric patients (aged $<18$ years) have not been established. ${ }^{3}$

\section{Transition to oral}

In cases where a transition to oral medication is desirable, clevidipine discontinuation or downward titration should take into account the time to onset of action of the oral therapy chosen. ${ }^{37}$

\section{Pharmacokinetics}

Clevidipine is practically insoluble in water and is available for clinical use as a lipid emulsion. ${ }^{14}$ Its half-life increases with lower blood temperature, and it is highly protein-bound in human plasma $(\sim 99.7 \%) .{ }^{38}$ In the perioperative setting, clevidipine butyrate produced a $4 \%-5 \%$ decrease in SBP within 2-4 minutes after the initiation of an infusion at a dose of $1-2 \mathrm{mg} / \mathrm{h} .{ }^{37}$ The time to onset of hemodynamic effects of clevidipine in healthy subjects was found to be within 2 minutes after start of infusion, and clevidipine reached arterial blood steady-state concentrations quickly. ${ }^{20}$

The action of clevidipine is independent of renal or hepatic functional status. Similar to esmolol, it is rapidly hydrolyzed by esterases in the blood and tissues $^{31}$ to a hemiacetal ester and butyric acid. ${ }^{9,20}$ These metabolites are subsequently metabolized to a large extent by decarboxylation before excretion through urine and fecal pathways. ${ }^{39}$ The same metabolites are formed after felodipine administration. ${ }^{9}$ Clevidipine has a mean blood flow clearance of $0.105 \mathrm{~L} / \mathrm{kg} / \mathrm{min}$ and a volume of distribution of $0.51 \mathrm{~L} / \mathrm{kg} .{ }^{1}$ Approximately $90 \%$ of R-clevidipine is cleared in 8 minutes and $90 \%$ of S-clevidipine is cleared in 11 minutes. ${ }^{40} \mathrm{~A}$ lag time of 0.5-1.0 minutes was observed between the termination of clevidipine infusion and the start of a rapid decay of clevidipine blood concentrations in venous blood samples collected from healthy volunteers and patients with essential hypertension. ${ }^{41}$ No lag time was observed in arterial blood samples from patients undergoing $\mathrm{CABG}$ surgery. ${ }^{42}$ Ericson et $\mathrm{l}^{43}$ reported that $\mathrm{BP}$ and heart rate return to predose values after discontinuation of the infusion within 10 minutes in healthy volunteers. 


\section{Contraindications}

Clevidipine is contraindicated in patients with allergies to soybeans, soy products, eggs, or egg products; patients with defective lipid metabolism (pathological hyperlipidemia, lipoid nephrosis, or acute pancreatitis, if it is accompanied by hyperlipidemia); and patients with severe aortic stenosis, in whom clevidipine may reduce myocardial oxygen delivery due to afterload reduction. ${ }^{37}$

\section{Precautions and side effects}

Clevidipine butyrate contains phospholipids that can support bacterial growth. Therefore, the vial must be changed every 4 hours once punctured. ${ }^{6}$ Doses should be adjusted for elderly patients, starting at the low end of the dosing range. In clinical trials, no differences in safety or effectiveness have been observed in patients older than 65 years compared with younger patients. Clevidipine may be initiated at $1-2 \mathrm{mg} / \mathrm{h}$ in patients with moderate to severe renal impairment and in patients with abnormal hepatic function, defined as one or more of the following: elevated serum bilirubin, aspartate aminotransferase/serum glutamic oxaloacetic transaminase, or alanine aminotransferase/serum glutamic pyruvic transaminase. ${ }^{3,37}$

Clevidipine is classified in Pregnancy Category C. Its effectiveness and safety have not been established in patients younger than 18 years. $^{3}$

\section{Cardiovascular side effects}

Clevidipine may cause systemic hypotension and reflex tachycardia. Patients who receive prolonged infusions of the new drug and are not switched to other antihypertensive agents should be monitored for rebound hypertension for at least 8 hours after discontinuation of the infusion. ${ }^{44}$ Patients with heart failure should be monitored closely because clevidipine, like other dihydropyridine CCBs, can produce negative inotropic effects. ${ }^{37}$

During Efficacy Study of Clevidipine Assessing its Postoperative Antihypertensive Effect in Cardiac Surgery-2 (ESCAPE-2) trial, a randomized, parallel, placebo-controlled, multicenter trial in cardiac surgery patients undergoing CABG (with or without valve replacement), atrial fibrillation was reported in $21 \%$ of patients with postoperative hypertension receiving intravenous clevidipine butyrate (over 30-60 minutes) compared with $12 \%$ of patients receiving placebo. No preoperative hypertension patients in The Efficacy Study of Clevidipine Assessing Its Preoperative Antihypertensive Effect in Cardiac Surgery (ESCAPE-1) trial receiving clevidipine butyrate or placebo experienced atrial fibrillation. ${ }^{44}$ Cardiac arrest and myocardial infarction were reported in less than $1 \%$ of patients in the ECLIPSE trial., 3,37

\section{Renal side effects}

During ESCAPE-1 trial, a randomized, parallel, placebo-controlled, multicenter trial in cardiac surgery patients undergoing CABG (with or without valve replacement), acute renal failure was reported in $9 \%$ of patients with preoperative hypertension receiving intravenous clevidipine butyrate (over 30 minutes, until treatment failure or anesthesia induction occurred) compared with $2 \%$ of patients receiving placebo. Acute renal failure was not reported among patients with postoperative hypertension receiving clevidipine butyrate (over 30-60 minutes) or in patients receiving placebo during the ESCAPE- 2 trial. ${ }^{37}$

\section{Therapeutic uses}

Clevidipine butyrate injectable emulsion is indicated for the reduction of BP when oral therapy is not feasible or desirable. $^{31}$

\section{Therapeutic efficacy in acute severe hypertension, the VELOCITY trial}

In the VELOCITY trial (EValuation of the Effect of ULtra-ShOrt-Acting Clevidipine in the Treatment of Patients with Severe HYpertension), Pollack et $\mathrm{al}^{2}$ evaluated the effect of ultrashort-acting clevidipine in the treatment of 131 patients with severe hypertension, defined as a SBP $>180 \mathrm{mmHg}$ and/ or DBP $>115 \mathrm{mmHg}$, with or without end-organ injury. This trial was performed as a multicenter, open-label, single-arm study to assess the efficacy and safety of intravenous clevidipine when initiated at a dose of $2 \mathrm{mg} / \mathrm{h}$.

Patients who had been treated with an antihypertensive within 2 hours of the study or who were unlikely to tolerate intravenous antihypertensive treatment of $>18$ hours duration were excluded, as were those with cirrhosis, liver failure, aortic dissection, or acute hypertension caused by use of, or withdrawal from, illicit drugs or alcohol, or an overdose. Clevidipine was administered via intravenous infusion to patients in the emergency department, an associated observation unit, or in ICU or coronary care units. ${ }^{2}$ The initial rate of administration was $2 \mathrm{mg} / \mathrm{h}$ for at least 3 minutes. After the initial titration, the agent was titrated on the basis of its efficacy, with a maximum infusion rate of $32 \mathrm{mg} / \mathrm{h}$, until SBP reached the predetermined target range. Patients continued to receive clevidipine for 18-96 hours if SBP reached the target range within the initial 30-minute treatment period. If target range was not achieved during the initial $30 \mathrm{~min}$ utes, the use of additional intravenous antihypertensive agents was permitted with or without clevidipine. The mean total dose of clevidipine infused during the study was $206 \mathrm{mg} .{ }^{2}$

Clevidipine was shown to be a safe and effective medication for rapidly reducing severely increased BP and controlling 
BP for 18 hours or longer. The results indicated that in an emergency department setting, an initial nonweight-based dose of $2 \mathrm{mg} / \mathrm{h}$ is appropriate. ${ }^{2,3}$ The proportion of patients whose initial target SBP was achieved within the initial 30 minutes of starting the clevidipine infusion was defined as the primary efficacy end point. The proportion of patients whose SBP was reduced to below the lower limit of the initial target range within 3 minutes of the drug infusion (ie, overshoot hypotension) was defined as a primary safety end point. Successful transition to oral antihypertensive, defined as maintenance of SBP within the target range for 6 hours after clevidipine discontinuation, was a secondary end point. ${ }^{2,3}$

A SBP target range (spanning $10-40 \mathrm{mmHg}$ ) was determined for each eligible patient. The initial mean SBP target range was $142.9-174.7 \mathrm{mmHg}$. Approximately 90\% of patients reached their SBP target within 30 minutes (the primary efficacy end point), which persisted for a minimum of 18 hours. The median time required to achieve target SBP was 9.5 minutes, consistent with other studies including ESCAPE-1 and -2 trials, which evaluated clevidipine compared with placebo in preoperative and postoperative cardiac surgery patients. ${ }^{2}$ Five patients had a prespecified SBP target range wider $(>40 \mathrm{mmHg})$ or narrower $(<20 \mathrm{mmHg})$ than the defined protocol. Only 2 patients $(1.6 \%)$ had SBP fall below the lower limit of the target range during the first 3 minutes of clevidipine infusion (primary safety end point). Among patients eligible for transition to oral therapy, $91.3 \%$ were successfully transitioned (secondary end point). ${ }^{3}$

Mean changes in SBP remained relatively steady throughout the drug infusion period (up to 60 hours), without increasing doses of clevidipine. Increase in baseline pulse rate was observed only during the first 15 minutes of infusion. ${ }^{2}$ The most common adverse effects reported were headache $(6.3 \%)$, nausea $(4.8 \%)$, chest discomfort (3.2\%), and vomiting $(3.2 \%)$. ${ }^{2,37}$

\section{Perioperative hypertension, the ECLIPSE, ESCAPE- I, and ESCAPE-2 trials}

Clevidipine was found to be comparably effective to nitroglycerin or sodium nitroprusside for the treatment of perioperative acute hypertension, or to nicardipine for postoperative hypertension, in patients undergoing cardiac surgery in the Evaluation of Clevidipine in the Perioperative Treatment of Hypertension Assessing Safety Events (ECLIPSE) trial. ${ }^{12}$ In the ESCAPE-1 trial, clevidipine was comparably effective for the treatment of preoperative hypertension in patients undergoing cardiac surgery compared with $20 \%$ lipid placebo emulsion. ${ }^{32}$ In the ESCAPE-2 trial, clevidipine was comparably effective for the treatment of postoperative hypertension in patients undergoing cardiac surgery compared with $20 \%$ lipid placebo emulsion. ${ }^{45}$

\section{Clevidipine vs placebo in ESCAPE trials}

The randomized, double-blind, placebo-controlled clinical trials (ESCAPE trials) demonstrated that clevidipine was safe and effective in controlling preoperative and postoperative hypertensions in hypertensive cardiac surgery patients compared with placebo..$^{32,45}$

Both preoperatively ${ }^{32}$ and postoperatively, ${ }^{45}$ the mean baseline BP did not differ significantly between the clevidipine and placebo groups in either ESCAPE trial (mean SBP $182 \mathrm{vs} 177 \mathrm{mmHg}$ and $147 \mathrm{vs} 151 \mathrm{mmHg}$, respectively). A history of previous myocardial infarction was more common in the clevidipine group, whereas angina pectoris and a family history of CAD were less common in clevidipine recipients. ${ }^{32}$ The majority ( $84 \%$ of patients) of both clevidipine and placebo recipients in ESCAPE-2 had a history of hypertension.

Patients received either intravenous clevidipine $0.5 \mathrm{mg} / \mathrm{mL}$ infused at an initial rate of $0.4 \mu \mathrm{g} / \mathrm{kg} / \mathrm{min}$ or placebo for at least 30 minutes $^{32,45}$ or until anesthesia was initiated, ${ }^{32}$ unless treatment failure occurred (primary end point: failure to reduce SBP by $\geq 15 \%$ from baseline or early and permanent discontinuation of treatment within 30 minutes of treatment initiation). ${ }^{32,45}$ Clevidipine was titrated as tolerated in doubling increments every 90 seconds up to $3.2 \mu \mathrm{g} / \mathrm{kg} / \mathrm{min}$. Infusion rates above $3.2 \mu \mathrm{g} / \mathrm{kg} / \mathrm{min}$ were based on patient response and were allowed in serial increments of $1.5 \mu \mathrm{g} / \mathrm{kg} / \mathrm{min}$. Infusion rates between 4.4 and $8 \mu \mathrm{g} / \mathrm{kg} / \mathrm{min}$ were administered for no longer than 2 hours, and no more than $500 \mathrm{~mL}$ of clevidipine infusion was administered in the first 24-hour period due to protocol-specified lipid-load restrictions..$^{37,45}$

Clevidipine was effective in reducing preoperative ${ }^{32}$ and postoperative $\mathrm{SBP}^{45}$ The treatment failure rate in the clevidipine group was $7.5 \%$, compared with $82.7 \%$ in the placebo group, with no observed lack of efficacy in the clevidipine group. ${ }^{32}$

The secondary outcome of time to reach the first measured reduction in SBP $\geq 15 \%$ from baseline was 6-8 minutes in the clevidipine group; the median time for the placebo group could not be estimated due to the small number of patients reaching the SBP target. ${ }^{32,45}$

The most common adverse effects reported in the clevidipine groups compared with placebo were pyrexia (18.9\% vs $13.7 \%$ ), atrial fibrillation ( $13.2 \%$ vs $11.8 \%$ ), acute renal insufficiency/failure (9.4\% vs $2 \%$ ), and nausea (5.7\% vs $9.8 \%$ ), respectively. One death occurred in the clevidipine group 
on postoperative day 1 , which was attributed to mediastinal hemorrhage and not to the study drug. . $^{32,37}$

\section{Clevidipine vs nicardipine hydrochloride, sodium nitroprusside, and nitroglycerin-the ECLIPSE trials}

The ECLIPSE study consisted of 3 parallel trials conducted at many medical centers where patients aged 18 years or older undergoing cardiac surgery were randomly assigned to receive clevidipine or a comparator drug, nitroglycerin, sodium nitroprusside, or nicardipine. ${ }^{12,45}$ Clevidipine was found to be comparably effective to nitroglycerin or sodium nitroprusside for the treatment of perioperative acute hypertension, and to nicardipine for postoperative hypertension, in patients undergoing cardiac surgery in these 3 prospective, randomized, open-label, parallel comparison trials.

During the initial 30 minutes of study, no other agents were permitted except in the case of treatment failure. If BP targets were not be achieved, alternative intravenous antihypertensive agent could be used. ${ }^{3}$ Study drug was primarily initiated during the preoperative or intraoperative period in the clevidipine vs nitroglycerin and clevidipine vs sodium nitroprusside groups. The majority of patients who received preoperative or intraoperative antihypertensive treatment also received clevidipine vs nicardipine in the postoperative setting per protocol, as the pharmacokinetic properties of nicardipine render it unsuitable for use in preoperative and intraoperative settings. ${ }^{12,45} \mathrm{BP}$ excursions were defined as the time and extent to which SBP exceeded or fell below individualized predetermined BP ranges. ${ }^{12}$

Clevidipine was initiated at an infusion rate of $0.4 \mu \mathrm{g} / \mathrm{kg} / \mathrm{min}$ and was titrated as tolerated in doubling increments every 90 seconds up to $3.2 \mu \mathrm{g} / \mathrm{kg} / \mathrm{min}$. Infusion rates above $3.2 \mu \mathrm{g} / \mathrm{kg} / \mathrm{min}$ were based on patient response and were allowed in serial increments of $1.5 \mu \mathrm{g} / \mathrm{kg} / \mathrm{min}$. Infusion rates between 4.4 and $8 \mu \mathrm{g} / \mathrm{kg} /$ min were used for no longer than 2 hours, and no more than 500 $\mathrm{mL}$ of clevidipine was administered in the first 24 -h period due to protocol-specified lipid-load restrictions. ${ }^{12,37}$

No differences were observed between patients receiving clevidipine compared with nitroglycerin, sodium nitroprusside, or nicardipine in the primary outcome of the 30-day incidence of death, stroke, myocardial infarction, or renal dysfunction. However, clevidipine was more effective at keeping BP within the prespecified range. ${ }^{37}$ Clevidipine was consistently predictable in controlling BP (with almost half of the BP excursions of the other agents; $3.8 \mathrm{vs}$ $7.8 \mathrm{mmHg} \times \mathrm{min} / \mathrm{h}$ ) because its fast onset and offset of action allowed for easy titration to maintain the target BP range in cardiac surgery patients with acute hypertension. ${ }^{12}$

The most commonly reported adverse events included atrial fibrillation and sinus tachycardia, which were similar in incidence for clevidipine and the comparator drugs. Atrial fibrillation was reported at $33.6 \%$ vs $32 \%$ (clevidipine vs nitroglycerin), $36.1 \%$ vs $32.2 \%$ (clevidipine vs sodium nitroprusside), and $35.6 \%$ vs $35.2 \%$ (clevidipine vs nicardipine). ${ }^{12,37,39}$

\section{Conclusion}

The rapid recognition and initiation of therapy are keys to minimize end-organ damage in patients with hypertensive emergency. Clevidipine is a vasoselective, ultrashort-acting, dihydropyridine L-type calcium channel antagonist with a rapid onset and offset of action, indicated for the reduction of BP when oral therapy is not feasible or desired. Clevidipine butyrate is an injectable emulsion, with pharmacokinetic and pharmacological properties that are well suited for acute BP control. Clevidipine has been shown to induce dose- and concentration-dependent decreases in mean arterial BP and SVR, with a little effect on heart rate and no effect on venous capacitance vessels.

Clevidipine has been shown to be a safe and effective medication for rapidly reducing severely increased BP and controlling BP for 18 hours or longer in emergency departments or associated observation units, or in ICU or coronary care units. Studies demonstrated that clevidipine was comparably effective to nitroglycerin or sodium nitroprusside for the treatment of perioperative acute hypertension, or to nicardipine for postoperative hypertension, in patients undergoing cardiac surgery.

\section{Disclosures}

The authors report no conflicts of interest in this work.

\section{References}

1. Varon J. Treatment of acute severe hypertension current and newer agents. Drugs. 2008;68(3):283-297.

2. Pollack C, Varon J, Garrison N, Ebrahimi R, Dunbar L. Clevidipine, an intravenous dihydropyridine calcium channel blocker, is safe and effective for the treatment of patients with acute severe hypertension. Ann Emerg Med. 2009;53(3):329-338.

3. Deeks E, Keating G, Keam S. Clevidipine: a review of its use in the management of acute hypertension. Am J Cardiovasc Drugs. 2009;9(2):117-134.

4. Potter JF. Malignant hypertension in the elderly. $Q J$ Med. 1995;88: 641-647.

5. National Heart, Lung, and Blood Institute. Seventh report of the Joint National Committee on prevention, detection, evaluation, and treatment of high blood pressure (JNC-7) 2003. Publication No. NIH 03-5233. Bethesda (MD): NIH; 2003.

6. Rhoney D, Peacock F. Intravenous therapy for hypertensive emergencies, part 1. Am J Health Syst Pharm. 2009;66:1343-1352.

7. Zampaglione B, Pascale C, Marchisio M, Cavallo-Perin P. Hypertensive urgencies and emergencies. Prevalence and clinical presentation. Hypertension. 1996;27:144-147. 
8. Slama M, Modeliar S. Hypertension in the intensive care unit. Curr Opin Cardiol. 2006;21:279-287.

9. Rodriguez G, Varon J. Clevidipine: a unique agent for the critical care practitioner. Crit Care Shock. 2006;9(2):37-41.

10. Marik PE, Varon J. Hypertensive crises: challenges and management. Chest. 2007;131:1949-1962.

11. Chobanian AV, Bakris GL, Black HR, et al. Seventh report of the Joint National Committee on prevention, detection, evaluation, and treatment of high blood pressure. Hypertension. 2003;42:1206-1252.

12. Aronson S, Dyke C, Stierer K, et al. The ECLIPSE trials: comparative studies of clevidipine to nitroglycerin, sodium nitroprusside, and nicardipine for acute hypertension treatment in cardiac surgery patients. Anesth Analg. 2008;107(4):1110-1121.

13. Bailey J, Lu W, Levy J, et al. Clevidipine in adult cardiac surgical patients a dose-finding study. Anesthesiology. 2002;96:1086-1094.

14. Prlesi L, Cheng-Lai A. Clevidipine: a novel ultra-short-acting calcium antagonist. Cardiol Rev. 2009;17:147-152.

15. Feldstein C. Management of hypertensive crises. Am J Ther. 2007; 14(2):135-139.

16. Weant KA, Flynn JD, Smith KM. Postoperative hypertension. Orthopedics. 2004;27:1159-1161.

17. Dasta JF, Bollinger JE, Gerlach AT, et al. National survey of acute hypertension management. Crit Care Med. 2007;35(12 Suppl):A89.

18. Desai R, Muntazar M, Goldberg M. Strategies for managing perioperative hypertension. Curr Hypertens Rep. 2009;11:173-177.

19. Murphy C. Hypertensive emergencies. Emerg Med Clin North Am. 2005; 13:973-1007.

20. Nordlander M, Sjoquist O, Ericsson H, et al. Pharmacodynamic, pharmacokinetics and clinical effects of clevidipine, a short acting calcium antagonist rapid blood pressure control. Cardiovasc Drug Rev. 2004;22:227-250.

21. Van Zwieten PA, van Wezel HB. Antihypertensive drug treatment in the perioperative period. J Cardiothorac Vasc Anesth. 1993;7:213-226.

22. Grossman E, Messerli FH. Calcium antagonists. Prog Cardiovasc Dis. 2004;47:34-57.

23. Fugit MD, Rubal BJ, Donovan DJ. Effects of intracoronary nicardipine, diltiazem and verapamil on coronary blood flow. J Invasive Cardiol. 2000; $12: 80-85$

24. Levy H. The ideal agent for perioperative hypertension and potential cytoprotective effects. Acta Anaesthesiol Scand. 1993;37:20-25.

25. Richard S. Vascular effects of calcium channel antagonists: new evidence. Drugs. 2005;65(2 Suppl):1-10.

26. Segawa D, Sjoquist O, Nordlander M, Wang QD, Gonon A, Ryden L. agd clevidipine, a new ultrashort-acting dihydropyridine. Eur J Pharmacol. 1999;380(2-3):123-128.

27. Nordlander M, Bjorkman JA, Regardh CG, Thalen P. Pharmacokinetics and hemodynamic effects of an uItrashort-acting calcium antagonist. Br J Anaesth. 1996;76(1):A24.

28. Goyal M, Jandhyala BS. Splanchnic artery occlusion shock (SAO) in hemodynamics. FASEB Exp Biol. 1997;97:A285.

29. Gourine A, Gonon A, Sjoquist P, et al. Short-acting calcium antagonist clevidipine protects against reperfusion injury via local nitric oxiderelated mechanisms in the jeopardized myocardium. Cardiovasc Res. 2001;51:100-107. Cardiac inotropic vs chronotropic selectivity of isradipine, nifedipine. the rats. Effect of clevidipine and sodium nitroprusside on splanchnic

30. Nguyen H, Ma K, Pham D, Pharm D. Clevidipine for the treatment of severe hypertension in adults. Clin Ther. 2010;32(1):11-23.

31. Cada D, Levien T, Baker D. Clevidipine butyrate injectable emulsion. Hosp Pharm. 2008;43(11):903-912.

32. Levy J, Mancao M, Gitter R, et al. Clevidipine effectively and rapidly controls blood pressure preoperatively in cardiac surgery patients: the results of the randomized, placebo-controlled efficacy study of clevidipine assessing its preoperative antihypertensive effect in cardiac surgery-1. Anesth Analg. 2007;105:918-925.

33. Schwieler H, Ericsson H, Lofdahl P, Thulin T, Kahan T. Circulatory effects and pharmacology of clevidipine, a novel ultra short acting and vascular selective calcium antagonist, in hypertensive humans. J Cardiovasc Pharmacol. 1999;34(2):268-274.

34. Eagle K, Berger P, Calkins H, et al. ACC/AHA guideline update for perioperative cardiovascular evaluation for noncardiac surgery executive summary. A report of the American College of Cardiology/ American Heart Association. Task force on practice guidelines comtask force on practice guidelines (committee to update the 1996 guidelines on perioperative cardiovascular evaluation for noncardiac surgery). Circulation. 2002;105:1257-1267.

35. Varon J, Marik PE. Clinical review: the management of hypertensive crises. Crit Care. 2003;7:374-384.

36. Varon J, Peacock W, Garrison N, et al. Prolonged infusion of clevidipine results in safe and predictable blood pressure control in patients with acute severe hypertension. Chest. 2007;132(4 Suppl):477.

37. Product Information: CLEVIPREX(R) IV Injection, Clevidipine Butyrate IV Injection. Clayton (NC): Hospira, Inc.; 2008.

38. Ericsson $\mathrm{H}$, Tholander B, Regardh CG. In vitro hydrolysis rate and protein binding of clevidipine, a new ultrashort-acting calcium antagonist metabolized by esterases in different animal species and man. Eur JPharm Sci. 1999;8(1):29-37.

39. Peacock W, Angeles J, Soto K, Lumb P, Varon J. Parenteral clevidipine for the acute control of blood pressure in the critically ill patient: a review. Ther Clin Risk Manag. 2009;5:627-634.

40. Ericsson H, Schwieler J, Lindmark BO, Lofdahl P, Thulin T, Regardh CG. Enantioselective pharmacokinetics of the enantiomers of clevidipine following intravenous infusion of the racemate in essential hypertensive patients. Chirality. 2001;13:130-134.

41. Ericsson H, Fakt C, Hoglund L, et al. Pharmacokinetics and pharmacodynamics of clevidipine in healthy volunteers after intravenous infusion. Eur J Clin Pharmacol. 1999;55(1):61-67.

42. Vylsteke A, Milner Q, Ericsson H, et al. Pharmacokinetics and pulmonary extraction of clevidipine, a new vasodilating ultrashort-acting dihydropyridine, in cardiac surgical patients during cardiopulmonary bypass. Br J Anaesth. 2000;85:683-689.

43. Ericsson H, Bredberg U, Enksson U, Jolin-Mellgard A, Nordlander M, Regardh CG. Pharmacokinetics and arteriovenous differences in clevidipine concentration following a short- and a long-term intravenous infusion in healthy volunteers. Anesthesiology. 2000;92(4):993-1001.

44. Hussar AD. New therapeutic agents marketed in the second half of 2008. Pharm Today. 2009;15(2):37-48.

45. Singla N, Warltier D, Gandhi S, et al. Treatment of acute postoperative hypertension in cardiac surgery patients: an Efficacy Study of Clevidipine Assessing its Postoperative Antihypertensive Effect in Cardiac Surgery-2 (ESCAPE-2), a randomized, double-blind, placebo-controlled trial. Anesth Analg. 2008;107(1):59-67.

\section{Dovepress}

\section{Publish your work in this journal}

Vascular Health and Risk Management is an international, peerreviewed journal of therapeutics and risk management, focusing on concise rapid reporting of clinical studies on the processes involved in the maintenance of vascular health; the monitoring, prevention and treatment of vascular disease and its sequelae; and the involvement of

metabolic disorders, particularly diabetes. This journal is indexed on PubMed Central and MedLine. The manuscript management system is completely online and includes a very quick and fair peer-review system, which is all easy to use. Visit http://www.dovepress.com/ testimonials.php to read real quotes from published authors. 